\section{nature research} EDITING SERVICE

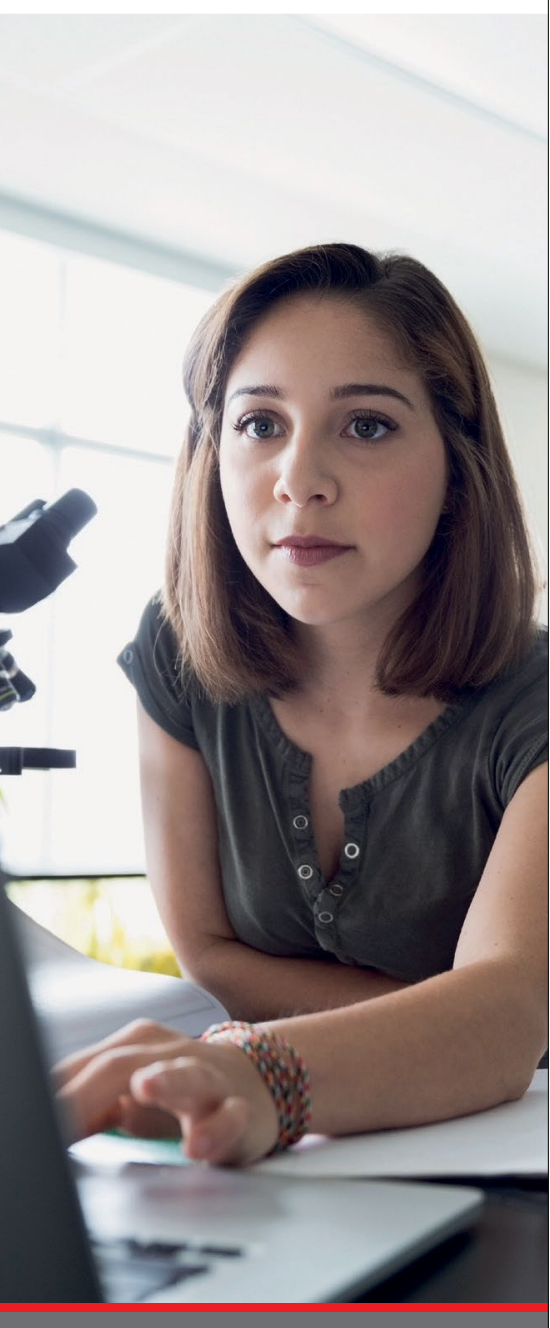

Could you communicate

your research more

effectively?

Our editors understand

what it takes to get

published and can offer

expert advice to help you

optimise your research

paper or grant proposal.

$\Rightarrow$ Learn more at

authorservices.

springernature.com/

scientific-editing

\section{MICROBIOLOGY}

\section{Measuring active microbiomes}

Couradeau, E. et al. Nat. Commun. 10, 2770 (2019).

Recent studies suggest that in soil samples, microbial taxa could possibly be recovered from extracellular DNA or dead cells. Thus, the link between soil processes and microbial composition can be misleading because of the presence of inactive cells. Methods such as BrdU labeling of DNA have been used to measure active microorganisms; however, these usually require extensively labeled DNA for sequencing. Couradeau et al. extended bioorthogonal noncanonical amino acid tagging (BONCAT) to probe translationally active cells. Specifically, soil samples are incubated with homopropargylglycine, which is incorporated into newly synthesized proteins and subsequently conjugated with fluorescent dyes via click chemistry. They then used fluorescence-activated cell sorting to recover BONCAT-positive cells and carried out 16S rRNA-seq analysis for the active community. They compared the active fraction extracted from two soil depths from Oak Ridge, TN, USA, and observed that the phylogenetic composition varied from the active fraction to the total population of extractable cells.

https://doi.org/10.1038/s41592-019-0520-x

\section{MICROSCOPY}

\section{Analyzing colocalization in SMLM}

Levet, F. et al. Nat. Commun. 10, 2379 (2019).

Single-molecule localization microscopy (SMLM) is becoming increasingly useful for quantitative analysis of cellular structures. Multicolor localization microscopy allows researchers to determine the relative positions of multiple structures, but parameters such as the degree of colocalization can be challenging to quantify, especially in 3D. Levet et al. have developed Coloc-Tesseler for analyzing colocalization in 2D and 3D SMLM data. Coloc-Tesseler begins by generating Voronoï diagrams for each color channel for image segmentation, which then enables standard colocalization metrics such as Manders and Pearson's coefficients to be calculated. Coloc-Tesseler's performance was validated on synthetic and experimental data, and it is likely to be a powerful tool for analyzing multicolor localization microscopy data. The software is implemented in a user-friendly GUI. $R S$

https://doi.org/10.1038/s41592-019-0521-9

\section{NEUROSCIENCE}

\section{A deeply learned brain atlas}

Iqbal, A. et al. Nat. Mach. Intell. 1, 277-287 (2019).

Chen, Y. et al. Nat. Methods 16, 341-350 (2019).

Segmenting and registration of brain imaging datasets can be a tedious and time-consuming task. Iqbal et al. now use a deep learning approach, which they call SeBRe, to facilitate this task for Nissl-stained, fluorescence, and even magnetic resonance image datasets. They trained a deep neural network to segment and classify eight different regions in the mouse brain. After training, SeBRe could segment and register other datasets with a precision of 0.84 , and similar performance could be achieved even if the brains were stained for previously unseen markers or imaged with a different microscopy modality. While SeBRe registers the image datasets to an existing brain atlas such as the Allen Brain Atlas, Chen et al. went a step further. They used convolutional neural networks to build a mouse brain atlas from scratch. This brain atlas is active and can be augmented with additional datasets. Furthermore, it preserves information on the variance between datasets. Both pipelines automate the processing of anatomical brain datasets and should substantially speed up the mapping of neurons and brain regions.

https://doi.org/10.1038/s41592-019-0522-8

\section{GENOMICS}

\section{Single-cell ChIP}

Grosselin, K. et al. Nat. Genet. 51, 1060-1066 (2019).

Rare cell types can have an outsized biological effect, but their sparsity makes them impossible to detect in bulk samples. To study the effect of such heterogeneity, Grosselin et al. developed a microfluidic device for single-cell chromatin immunoprecipitations. In one channel, cells are embedded in droplets, lysed and treated with MNase to fragment their chromatin; in another channel, barcoded beads are encapsulated. Following fusion of these two types of droplets, the nucleosomes in each cell are tagged with a specific barcode. After pooling and immunoprecipitation of activating and repressing histone marks, each modification can be traced back to its cell of origin. The authors probed hundreds of cells from drugresistant and susceptible breast tumors, and found a resistance signature in a low percentage of drug-sensitive cells. At $4 \%$ of the overall population, this cell type was too sparse to be detected in a bulk sample, but it can provide important clues about the development of drug resistance during tumor progression. NR

https://doi.org/10.1038/s41592-019-0523-7

Nicole Rusk, Arunima Singh, Rita Strack, Lei Tang and Nina Vogt 